\title{
BMJ Open Effect of single family rooms for preterm infants on neurodevelopment: study protocol for a systematic review
}

\author{
Nicole R van Veenendaal, ${ }^{1,2}$ Sophie R D van der Schoor, ${ }^{1}$ Jacqueline Limpens, ${ }^{3}$ \\ Anne A M W van Kempen, ${ }^{1}$ Johannes B van Goudoever ${ }^{2,4}$
}

To cite: van Veenendaal NR, van der Schoor SRD, Limpens J, et al. Effect of single family rooms for preterm infants on neurodevelopment: study protocol for a systematic review. BMJ Open 2017;7:e015818. doi:10.1136/ bmjopen-2017-015818

- Prepublication history and additional material for this paper are available online. To view these files please visit the journal online (http://dx.doi. org/10.1136/bmjopen-2017015818).

Received 5 January 2017 Revised 20 March 2017 Accepted 4 May 2017

\section{CrossMark}

${ }^{1}$ Department of Pediatrics/ Neonatology, OLVG location East, Amsterdam, The Netherlands ${ }^{2}$ Department of Pediatrics, Emma Children's Hospital Academic Medical Center, Amsterdam, The Netherlands ${ }^{3}$ Medical Library, Academic Medical Center, Amsterdam, The Netherlands

${ }^{4}$ Department of Pediatrics, VU University Medical Center, Amsterdam, The Netherlands

Correspondence to Nicole $R$ van Veenendaal; n.r.vanveenendaal@amc.uva.nl, n.r.vanveenendaal@olvg.nl

\section{ABSTRACT}

Introduction Preterm infants are at an increased risk for neurodevelopmental delay. They have to endure many stressors in early life, including parent-infant separation, noise and painful procedures during hospitalisation in the highly technological environment of the modern neonatal ward. Currently, a shift is being noticed in the architectural design of neonatal wards towards single family rooms instead of the common open bay units. The influence of the hospital environment on health and specifically neurodevelopment in this vulnerable patient population remains under discussion.

Objectives To assess the effect of single family rooms during hospitalisation primarily on neurodevelopment in preterm infants. Secondary outcome measures will be neonatal (ie, breastfeeding rates, sepsis, growth during hospital stay, length of hospital stay) and parental (ie, parental stress, satisfaction, participation, presence and self-efficacy).

Methods and analysis The PRISMA-P 2015 (Preferred Reporting Items for Systematic reviews and Meta-Analyses for Protocols 2015) 17 items checklist was used for the generation of the protocol for this review. The following PICO was formulated: Population: preterm infants with need of hospitalisation in the neonatal ward; Intervention: single family rooms; Comparison: standard neonatal care in open bay units; Outcome: neurodevelopmental outcome of infants from 9 months onwards. If at least two studies, with low or moderate risk of bias, suitable for inclusion are found a meta-analysis will be performed. If quantitative synthesis is not appropriate the data will be presented descriptively.

Dissemination plans This will be the first review, systematically assessing the effect of single family rooms on neurodevelopmental outcome in preterm infants. Clinical practice could possibly be optimised to ameliorate neurodevelopment in this vulnerable patient population based on these insights. This systematic review will be published in an international peerreviewed journal.

Registration We registered this systematic review protocol with the PROSPERO (International Prospective Register of Systematic Reviews) on 2 November 2016 (registration number: CRD42016050643).

Ethics We will use data from patients enrolled in studies and/or trials already approved by the relevant ethical committees and therefore this systematic review requires no further permissions.

\section{Strengths and limitations of this study}

- This systematic review and meta-analysis will represent, to our knowledge, the most comprehensive analysis of the evidence on single family rooms and neurodevelopment for preterm infants.

- The strengths include the systematic and detailed search strategy, advanced registration and dissemination of the review strategy; we used explicit inclusion and exclusion criteria and described duplicate independent screening, data extraction and risk of bias assessment.

- The study was limited by the protocol phase and therefore no results are yet presented; our final conclusions may be limited by the number and quality of available studies and clinical, methodological or statistical heterogeneity.

- With this systematic review we may detect small or moderate, but potentially meaningful differences, that individual trials were not able to establish.

- This prospectively defined protocol enhances the expected utility and applicability of the final results to ultimately influence neonatal practice.

\section{INTRODUCTION}

Background and rationale

An estimated 15 million babies are born preterm annually, $11.1 \%$ of all live births worldwide. This percentage ranges from about $5 \%$ in several European countries to $18 \%$ in some African countries, and it is rising. ${ }^{2}$ Direct complications of preterm birth account for 1 million deaths each year, and preterm birth is a risk factor in over $50 \%$ of all neonatal deaths. In addition, preterm birth can result in a range of longterm complications in survivors, with lifelong effects on metabolic, respiratory, neurological and physical health, which contribute to the prematurity-related burden of chronic disease in adulthood. ${ }^{3}$ Most notably, preterm infants are at increased risk for developmental difficulties. Cerebral palsy, attention deficit and hyperactivity, cognitive delays and 
executive functioning disorders may appear in spite of modern lifesaving technology. ${ }^{4}$

The advances in technology are increasing the survival of preterm infants, and the focus in caregiving for this vulnerable population shifts to decreasing the burden of chronic disease and improving the quality of life and neurodevelopmental outcome. Evidence is increasing that immaturity in itself and unfavourable environmental factors (such as separation of parents, lighting, noise, exposure to varying levels of pain and pain-related stress) during hospitalisation in the neonatal ward might influence the range of morbidities in preterm infants including neurodevelopment and psychosocial behaviour. ${ }^{5-7}$

Also, due to the technological environment and enhancement of the modern neonatal ward, premature or ill term newborns and their parents are commonly separated worldwide during hospitalisation, and both physical and emotional closeness is impaired. ${ }^{8}$ The early postnatal life is a sensitive period and impairment of mother-infant interactions (such as maternal separation or deprivation) during the early postnatal period has been shown to disrupt neuroendocrine regulations, such as upregulation of hippocampal glucocorticoid receptor and hypothalamic corticotropin-releasing factor, along with increased corticosterone and adrenocorticotropic hormone levels. ${ }^{10}$ These early stress-induced neuroendocrine alterations may be associated with behavioural problems in adulthood, such as impaired memory, learning and anxiety and depressive-like behaviours, next to prematurity in itself. ${ }^{10}$

Currently, a shift is being noticed in the field towards single family rooms (SFR) instead of the common open bay neonatal wards. Evidence in the Neonatal Intensive Care Unit (NICU) setting has shown that infants in the SFR setting weighed more at discharge, had a greater rate of weight gain, required fewer medical procedures, had a lower gestational age (GA) at full enteral feed and less sepsis, showed better attention, less physiologic stress, less hypertonicity, less lethargy and less pain. ${ }^{11}$ In conjunction, less maternal stress in the SFR NICU was present and some of the reduction in stress in the infants was related to increased maternal involvement. ${ }^{11}$ Mothers with newborns in SFR nurseries spent markedly more time with their newborns, as opposed to mothers of newborns in open bay unit (OBU) nurseries during the first 2 weeks of their newborns' lives. ${ }^{12}$ Mothers who roomed-in with their preterm infant were more likely to initiate breast feeding. ${ }^{13}$ At discharge from the hospital and at 4 months postdischarge mothers of newborns in SFR nurseries were significantly more likely to exclusively breast feed their newborns as compared with mothers of infants in OBU nurseries. ${ }^{12}$

A previous review focused on early interventions involving parents to improve neurodevelopmental outcomes in premature infants. Teaching parenting skills, and/or involving parents in the hospital care of the preterm appeared to be beneficial on long-term neurodevelopment up to an age of 36 months,${ }^{14}$ however this review did not include and/or assessed the influence of SFRs. Another review discussed the beneficial outcomes according to the design of neonatal intensive care unit solely focusing on the difference between open bay and SFRs in the NICU setting, with regard to published research between 2000 and $2011,{ }^{15}$ without assessing neurodevelopmental outcome. Several studies have assessed the influence of SFRs on neurodevelopmental outcome with contradictory results. ${ }^{16} 17$

\section{Description of the intervention}

\section{Single family rooms}

These are private rooms in which families are provided with the (architectural) accommodation to stay with their infant continuously, day and night, and to be able to provide daily care.

\section{How the intervention might work}

SFRs might facilitate parent-infant closeness, increasing possibilities to perform kangaroo-mother-care and interaction between parents and infants with increased privacy. ${ }^{5} 18$ Also, it may provide the type of high-quality care and physical environment that will lead to improved infant outcome through possible expression of specific genes in brain regions, reducing stress reactivity and better regulated infant behaviour. ${ }^{5}$ It might reduce sound and noise levels from the other infants otherwise accompanying them on a neonatal open bay ward, which have sensory and adverse influences. ${ }^{5} 1920$ It might improve sleeping patterns. ${ }^{5} 18$ Also, sepsis rates might be reduced, due to the physical separation between preterm infants and providing areas for hand washing within each room. ${ }^{5112122}$ With the implementation, SFRs might promote developmental care and family-centred care. ${ }^{5}$ Increasing closeness, reducing stressors and sepsis, increasing and encouraging possibly the length and amount of breast feeding might enhance or alter brain plasticity through experience and neurodevelopment of preterm infants.

\section{Why it is important to do this review}

Preterm infants are commonly cared for in open bay neonatal wards, though a shift is being noticed in the field towards SFRs. The influence of the hospital environment on health and specifically neurodevelopment of preterm infants remains under discussion. It is important to review the literature to examine the effect of SFRs on neurodevelopment in this vulnerable patient population and to possibly ameliorate caregiving practices for these individuals.

\section{Objectives}

\section{Primary objectives}

To assess the effect of SFRs in comparison to standard neonatal care in OBUs during hospitalisation on neurodevelopment in preterm infants.

The following PICO was formulated:

Population:

Preterm infants with need of hospitalisation in the neonatal ward 
Intervention:

Single family rooms

Comparison:

Standard neonatal care in OBUs

\section{Outcome:}

Neurodevelopmental outcome of infants from 9 months onwards

\section{Secondary objectives}

To assess the effect of SFRs in comparison to standard neonatal care in OBUs during hospitalisation on:

- Neonatal outcome measures: breastfeeding rates, sepsis, growth during hospital stay, length of hospital stay, mortality, morbidity (including retinopathy of prematurity (ROP), bronchopulmonary dysplasia (BPD) and necrotising enterocolitis (NEC)) critical incidents, cortisol levels and outcome measures defined in the papers to be of relevance.

- Parental stress, satisfaction, participation, self-efficacy, bonding and outcome measures defined in the papers to be of relevance.

\section{METHODS}

The PRISMA-P 2015 (Preferred Reporting Items for Systematic reviews and Meta-Analyses for Protocols 2015) 17 items checklist was used to generate the protocol for this review. ${ }^{23}$ In accordance with the recommendations, this protocol was registered with the PROSPERO (International Prospective Register of Systematic Reviews) on 2 November 2016 (registration number: CRD42016050643).

\section{Eligibility criteria}

Study designs

We will include investigational studies including randomised controlled trials (RCTs) or cluster-randomised trials. Also, we will examine studies with a quasi-randomised, controlled before-after (CBAs) or interrupted time series (ITS) design for this review. Observational studies, including cohort, case-control and cross-sectional studies with clear intervention and control participants will be considered for this review.

\section{Participants}

Preterm infants (GA $<37$ weeks) and their parents with need of hospitalisation in the neonatal ward due to the condition of the child. Inclusion criteria: infants include stable preterm infants. Stable infants are defined as preterm infants, without the need of haemodynamic stimulants, stable according to the attending physician on non-invasive respiratory support including continuous positive airway pressure (CPAP)/ nasal flow/low flow or without respiratory support. Infants are allowed to have tube feeding, parenteral feeding or oral feeding and central venous lines/catheters. No restrictions for birth weight are applied.

\section{Exclusion criteria}

Asphyxia defined as infants with American College of Obstetrics and Gynaecology (ACOG) criteria (umbilical
$\mathrm{pH}<7.00$ or Apgar score 3 or lower at $5 \mathrm{~min}$ ) will be excluded, since asphyxia has a major influence on neurodevelopmental outcome. ${ }^{24}$ Parents of infants with severe psychosocial disorders (mental illness requiring hospitalisation) or under supervision of youth care will be excluded. Children with congenital abnormalities will be excluded.

If studies of mixed participant samples are identified relevant to our review, study authors will be contacted to request the subgroup data for preterm participants only.

\section{Interventions}

The intervention is aimed at in-hospital stay of the infant without a communal component.

\section{Single family rooms}

SFRs are rooms, in which each single patient (the infant) has a private room, enabling the parents to be present continuously, also during the night. The room is equipped with all necessary medical and nursery care. Tube feeding, cardiorespiratory monitoring, respiratory support, antibiotics or phototherapy can be provided. It provides space for the parent(s) to sleep and be present continuously (including overnight). We will regard single patient rooms as equivalent to SFRs if parents can stay present continuously.

\section{Open bay units}

These units are OBUs with newborns staying communally in one room or open bay ward, often in close proximity to the maternity ward, but physically separated. Neonates who require high care, tube feeding, cardiorespiratory monitoring, respiratory support, antibiotics or phototherapy are admitted to these wards. Medical rounds are done in a separate room from the OBU, between the nurses and the doctors. Nurses provide routine care, and parents are welcome at all times. Due to the setting, the duration that parents can stay at the bedside of their infant is limited. OBUs do not provide 24-hour facilities, especially not a place to sleep or rest for the mother.

\section{Types of outcome measures}

\section{Primary outcomes}

We will include studies reporting the following outcomes: neurodevelopmental outcome of infants (from 9 months onwards). The main interest point are age-appropriate standardised scales, for instance the mental developmental index or psychomotor developmental index of the Bayley Scale of Infant Development (BSID, BSID-II or BSID-III). ${ }^{25}$ The following scales will also be considered for this review (as described before ${ }^{14}$ ): the McCarthy Scales of Children's Abilities, ${ }^{26}$ Stanford-Binet Intelligence Scale, ${ }^{27}$ the Griffiths Mental Development Scale ${ }^{28}$ and the Wechsler Preschool and Primary Scale of Intelligence. ${ }^{29}$ Definitions of outcomes will be extracted as reported in the individual studies. We will extract the outcomes (eg, ordinal, dichotomous, continuous) as reported in the included studies. 


\section{Timing}

Studies will be included if at least $70 \%$ of participants were followed up for neurodevelopment for at least 9 months.

\section{Secondary outcomes}

1. Neonatal outcome measures: for example, breastfeeding rates, sepsis, growth, length of hospital stay, mortality and morbidity (including ROP, BPD and NEC) critical incidents, cortisol levels, readmissions to hospital. Short-term neurodevelopmental outcome measures $(<9$ months of age) will be considered as secondary outcomes; for instance, the NICU Network Neurobehavioral Scale ${ }^{30}$ and the Neonatal Behavioral Assessment Scale. ${ }^{31}$

2. Parental stress, satisfaction, participation, presence, self-efficacy and parent-infant bonding as measured by standardised scales, for instance the parental stressor scale.

\section{Timing}

If feasible, we will make comparisons for the secondary objectives at different follow-up periods:

- during hospital stay

- short term: less than 3 months after hospital discharge

- long term: after 9 months after hospital discharge.

\section{Language}

Studies reporting in the English, Dutch or a language for which a translator is available will be included.

\section{Information sources}

\section{Electronic searches and search strategy}

An experienced medical information specialist (JL) will search the electronic databases MEDLINE, EMBASE, PsycINFO (OVID), CENTRAL (the Cochrane Central Register of Controlled Trials) and clinical trial registers. ${ }^{32}$ The electronic search strategy combines controlled terms (i.e., MeSH) and text words for preterm infants (including preterm diseases and NICU) and for terms indicating single (family) rooms. The search strategy will not be limited bystudy design,language or date. The current version of the MEDLINE search strategy is available in online supplementary file 1 .

\section{Searching other resources}

Two experts in the field will be contacted to examine a list of all relevant and related articles to determine any apertures or supplemental unpublished studies. We will consider conference and symposia abstracts in the English and Dutch languages and for which a translator is accessible for this review (until 31 December 2016). We will cross-check reference lists and cited articles of identified relevant papers for any related references.

\section{Data collection and analysis}

Selection of studies

The list of references will be downloaded, imported and deduplicated in ENDNOTE.

Two authors (NRvV and SRDvdS) will independently discern articles by screening titles and abstracts for relevance with respect to selection criteria using Rayyan ${ }^{33}$. Selection criteria are based on research setting, study design and reported results. Studies considering the design of the neonatal ward that did not describe specific design features (ie, SFRs, or single patient rooms, or OBUs) and the impact on outcome measures will be excluded. The reviewers will classify the abstracts as: included, uncertain or excluded.

Reviewers (NRvV and SRDvdS) will independently and systematically review full texts of selected abstracts (categorised as included or uncertain) for further eligibility. They will use a standardised form with clear inclusion and exclusion criteria. The two reviewers will resolve disagreement by discussion. If dissent persists between the two reviewers an independent epidemiologist and researcher will be consulted.

\section{Data extraction and management}

An explicit and standardised data collection form will be used by two reviewers ( $\mathrm{NRvV}$ and SRDvdS). The two reviewers will independently extract data from relevant studies, including but not restricted to items on study characteristics, confounding factors and possible cointerventions (items as listed in the PICO). The reviewers will accumulate the following items for this review: country in which the study was performed, design of the neonatal ward, parental presence, hours of kangaroo-mother/ skin-to-skin care, parental participation/engagement, concept of care given, year of publication, study design, methodology, sample size, duration of follow-up, definition of SFR used in study, measurement tool or method used to quantify neurodevelopment, confounders for neurodevelopment assessed, unit of measurement (if appropriate), number and/or times of follow-up measurements, number of participants included in analysis, number of withdrawals, exclusions, lost to follow-up, participants' characteristics (neonatal or parental) and all patient-important reported outcomes. RevMan V.5.3.5 will be used. If multiple publications report data from the same study population, the most recent outcome of interest will be assessed. We will get in touch with study authors twice when relevant data are not explicitly or clearly reported.

\section{Risk of bias in individual studies}

Risk of bias in randomised trials will be assessed independently by two reviewers ( $\mathrm{NRvV}$ and SRDvdS) using the Cochrane Collaboration's Risk of Bias Tool (assessing allocation concealment, blindness of intervention, completeness of follow-up, blinding of outcome assessment). To determine the quality of ITS, CBA studies, cohort, case-control studies and cross-sectional studies, the reviewers will independently use the Cochrane Risk of Bias Tool for Non-Randomised Studies of Interventions (ROBINS-I) ${ }^{34}$ We will calculate the kappa score for the risk of bias across the seven domains of the ROBINS-I tool. According to the methodological characteristics assessed, reviewers will independently categorise the studies' risk of bias as low, moderate, serious and 
critical. This will be done in duplicate. Disagreement will be resolved by discussion and then by consulting a third author.

\section{Data synthesis}

Measures of treatment effect: neurodevelopment

If the two reviewers independently find at least two studies, with low or moderate risk of bias, suitable for inclusion, with acceptable heterogeneity a meta-analysis will be performed. We will use a random-effects model to combine the results on neurodevelopment in a meta-analysis. Continuous data (eg, Bayley scales of development) will be analysed computing the weighted mean difference with $95 \%$ CIs (as described before ${ }^{14} 35$ ). The weighted mean difference is calculated by weighing the inverse of the variance of the non-standardised difference between the mean cognitive test scores of the preterm infants cared for with SFR and the preterm infants cared for in OBUs. Cognitive scores from all studies will be obtained from comparable tests of cognition; see also the Primary outcomes section. ${ }^{25-29}$ For dichotomous data we will calculate the effect size as ORs with their 95\% CIs. Where feasible, results will be shown graphically using a forest plot.

The direction of the effect, the size of the effect and the consistency of the effect of hospital setting on neurodevelopment in preterm infants will be assessed in the included studies. Review Manager (RevMan, V.5.3.5) will be used.

If quantitative synthesis is not appropriate we will not perform a meta-analysis and data will be presented descriptively. We will use guidance from the Centre for Reviews and Dissemination to provide full-text narrative review of our findings. ${ }^{36}$

\section{Dealing with missing data}

Original authors will be contacted twice to request missing data. We will use sensitivity analysis to analyse missing data, imputing the worst and best outcome as described in the paper of relevance.

\section{Assessment of heterogeneity}

Heterogeneity will be assessed computing $\mathrm{I}^{2}$ to quantify inconsistency and variability to be present in the meta-analysis. ${ }^{37}$ It will be considered as follows (as described in the Cochrane Handbook for Systematic Reviews of Interventions $^{38}$ ): 0\%-40\% might not be important; 30\%-60\% may represent moderate heterogeneity; 50\%-90\% may represent substantial heterogeneity; $75 \%-100 \%$ represents considerable heterogeneity. If substantial heterogeneity is present we will not perform a meta-analysis. Linear meta-analysis regression models will be used to explore the impact of covariates specified by the studies on heterogeneity.

\section{Subgroup analysis}

If sufficient studies are available, subgroup analyses will examine:

- The differential effect of SFR by extent of prematurity (eg, extremely preterm GA $<26$ weeks, very pre- term $<32$ weeks and late preterm $<37$ weeks of gestation).

A priori, we will define subgroups according to neonatal risk and type of intervention. High-risk neonates are defined as infants with brain structural abnormalities (including periventricular leucomalacia and intraventricular haemorrhage) or sensory abnormalities (ROP, blindness or deafness) or motor disabilities (including cerebral palsy) as described before. ${ }^{14}$ Infants without above criteria are defined as low-risk infants.

Only if more than 10 studies are available for meta-analysis, we will use meta-regression to estimate the effect of the different study designs with their individual risk of bias on outcome variables.

\section{Sensitivity analysis}

We will perform sensitivity analyses to assess if the findings are robust over the assumptions made during the review process.

Possible decisions which will be considered to be subject of the sensitivity analysis are: eligibility criteria, data analysed and analysis methods. Especially risk of bias will be subject to sensitivity analysis to analyse how conclusions might be affected if studies at high risk of bias were included. If SFRs without facilities for parents to be able to be present 24 hours/day are encountered, we will perform sensitivity analyses to estimate the influence of parental presence. Sensitivity analyses will be reported in a summary table.

\section{Metabias}

Funnel plots will be used to assess publication bias. The recommendations on testing for funnel plot asymmetry as described in the Cochrane Handbook for Systematic Reviews for Interventions will be followed. ${ }^{38}$

\section{Confidence in cumulative evidence}

We will use the Grading of Recommendations Assessment, Development and Evaluation working group methodology to assess the quality of evidence for all outcomes.

\section{Dissemination plans}

This will be the first review, which systematically assesses the effect of SFRs on neurodevelopmental outcome in preterm infants. With these insights clinical practice could possibly be optimised to ameliorate outcome and specifically neurodevelopment in this vulnerable patient population. This systematic review will be published in an international peer-reviewed journal.

Contributors NRvV, SRDvdS, AAMWvK and JBvG drafted the manuscript. All authors contributed to the development of the selection criteria, the risk of bias assessment strategy and data extraction criteria. NRVV developed a search strategy in collaboration with a medical information specialist (JL) with systematic review experience. SRDvdS, AAMWvK and JBvG provided expertise on single family rooms, neurodevelopment and prematurity. All authors equally read, provided feedback and approved the final manuscript.

Funding This research project received no specific grant from any funding agency in the public, commercial or not-for-profit sectors. NRvV is supported by an unrestricted research grant by Nutricia, The Netherlands.

Competing interests None declared.

Provenance and peer review Not commissioned; externally peer reviewed. 
Open Access This is an Open Access article distributed in accordance with the Creative Commons Attribution Non Commercial (CC BY-NC 4.0) license, which permits others to distribute, remix, adapt, build upon this work non-commercially, and license their derivative works on different terms, provided the original work is properly cited and the use is non-commercial. See: http://creativecommons.org/ licenses/by-nc/4.0/

(c) Article author(s) (or their employer(s) unless otherwise stated in the text of the article) 2017. All rights reserved. No commercial use is permitted unless otherwise expressly granted.

\section{REFERENCES}

1. Blencowe $\mathrm{H}$, Cousens $\mathrm{S}$, Oestergaard $\mathrm{MZ}$, et al. National, regional, and worldwide estimates of preterm birth rates in the year 2010 with time trends since 1990 for selected countries: a systematic analysis and implications. Lancet 2012;379:2162-72.

2. Blencowe H, Cousens S, Chou D, et al. Born Too Soon Preterm Birth Action Group. Born too soon: the global epidemiology of 15 million preterm births. Reprod Health 2013;10(Suppl 1):S2.

3. Blencowe H, Lee AC, Cousens S, et al. Preterm birth-associated neurodevelopmental impairment estimates at regional and global levels for 2010. Pediatr Res 2013;74 Suppl 1:17-34.

4. Pineda RG, Tjoeng TH, Vavasseur C, et al. Patterns of altered neurobehavior in preterm infants within the neonatal intensive care unit. J Pediatr 2013;162:470-6.

5. Lester BM, Miller RJ, Hawes K, et al. Infant neurobehavioral development. Semin Perinatol 2011;35:8-19.

6. Provenzi L, Giusti L, Fumagalli M, et al. Pain-related stress in the Neonatal Intensive Care Unit and salivary cortisol reactivity to socio-emotional stress in 3-month-old very preterm infants. Psychoneuroendocrinology 2016;72:161-5.

7. Ranger M, Zwicker JG, Chau CM, et al. Neonatal Pain and infection relate to smaller cerebellum in very Preterm Children at School Age. J Pediatr 2015;167:292-8.

8. Flacking R, Lehtonen L, Thomson G, et al. Separation and Closeness Experiences in the Neonatal Environment (SCENE) group. Closeness and separation in neonatal intensive care. Acta Paediatr 2012;101:1032-7.

9. Flacking R, Dykes F. 'Being in a womb' or 'playing musical chairs': the impact of place and space on infant feeding in NICUs. BMC Pregnancy Childbirth 2013;13:179.

10. Vaiserman AM. Epigenetic programming by early-life stress: evidence from human populations. Dev Dyn 2015;244:254-65.

11. Lester BM, Hawes K, Abar B, et al. Single-family room care and neurobehavioral and medical outcomes in preterm infants. Pediatrics 2014;134:754-60.

12. Jones R, Jones L, Feary AM. The effects of Single-Family Rooms on Parenting Behavior and Maternal psychological factors. J Obstet Gynecol Neonatal Nurs 2016;45:359-70.

13. Tavoulari EF, Benetou V, Vlastarakos PV, et al. Factors affecting breast-feeding initiation in Greece: What is important? Midwifery 2015;31:323-31.

14. Vanderveen JA, Bassler D, Robertson CM, et al. Early interventions involving parents to improve neurodevelopmental outcomes of premature infants: a meta-analysis. J Perinatol 2009;29:343-51.

15. Shahheidari M, Homer C. Impact of the Design of Neonatal Intensive Care Units on Neonates, Staff, and Families. J Perinat Neonatal Nurs 2012;26:260-6.

16. Lester BM, Salisbury AL, Hawes K, et al. 18-Month Follow-Up of Infants Cared for in a Single-Family Room Neonatal Intensive Care Unit. J Pediatr 2016;177:84-9.
17. Pineda RG, Neil J, Dierker D, et al. Alterations in brain structure and neurodevelopmental outcome in preterm infants hospitalized in different neonatal intensive care unit environments. J Pediatr 2014;164:52-60.

18. Johnson $\mathrm{BH}$, Abraham MR, Parrish RN. Designing the neonatal intensive care unit for optimal family involvement. Clin Perinatol 2004;31:353-82.

19. Glass P, Avery GB, Subramanian KN, et al. Effect of bright light in the hospital nursery on the incidence of retinopathy of prematurity. $N$ Engl J Med 1985;313:401-4.

20. American academy of Pediatrics Committee on Environmental Health. noise: a hazard for the fetus and newborn. Pediatrics 1997;100:724-7.

21. Andersen BM, Lindemann R, Bergh K, et al. Spread of methicillinresistant Staphylococcus aureus in a neonatal intensive unit associated with understaffing, overcrowding and mixing of patients. J Hosp Infect 2002;50:18-24.

22. Detsky ME, Etchells E. Single-patient rooms for safe patient-centered hospitals. JAMA 2008;300:954-6.

23. Moher D, Shamseer L, Clarke M, et al. PRISMA-P Group. Preferred reporting items for systematic review and meta-analysis protocols (PRISMA-P) 2015 statement. Syst Rev 2015;4:1-9.

24. Dilenge ME, Majnemer A, Shevell MI. Long-term developmental outcome of asphyxiated term neonates. J Child Neurol 2001;16:781-92.

25. Bayley N. Scales of infant development. New York: Psychologica Corp, 1969.

26. McCarthy D. McCarthy scales of children's abilities. New York: Psychological Corp, 1972.

27. Laurent J, Swerdlik M, Ryburn M. Review of validity research on the Stanford-Binet Intelligence Scale: Fourth Edition. Psychol Assess 1992;4:102-12.

28. Griffiths R. The ability of Young Children: a Comprehensive System of Mental Measurement for the First eight years of Life. Association for Research in Infant and Child Development 1984.

29. Koriakin TA, McCurdy MD, Papazoglou A, et al. Classification of intellectual disability using the Wechsler Intelligence Scale for Children: Full Scale IQ or General Abilities Index? Dev Med Child Neurol 2013:55:840-5.

30. Lester BM, Tronick EZ, Brazelton TB. The Neonatal Intensive Care Unit Network Neurobehavioral Scale procedures. Pediatrics 2004;113:641-67.

31. Brazelton TB, Nugent JK. Neonatal Behavioral Assessment Scale: Cambridge University Press, 1995.

32. Rethlefsen ML, Murad MH, Livingston EH. Engaging medical librarians to improve the quality of review articles. JAMA 2014;312:999-1000.

33 Ouzzani M, Hammady H, Fedorowicz Z, et al. Rayyan-a web and mobile app for systematic reviews. Syst Rev 2016;5:210-.

34 Sterne JA, Hernán MA, Reeves BC, et al. ROBINS-I: a tool for assessing risk of bias in non-randomised studies of interventions. BMJ 2016;355:i4919.

35 Bhutta AT, Cleves MA, Casey PH, et al. Cognitive and behavioral outcomes of school-aged children who were born preterm: a metaanalysis. JAMA 2002;288:728-37.

36 Centre for Reviews and Dissemination. systematic reviews: crd's guidance for undertaking reviews in health care. CRD, University of York 2009 https://www.york.ac.uk/crd/guidance/.

37 Higgins JP, Thompson SG. Quantifying heterogeneity in a metaanalysis. Stat Med 2002;21:1539-58.

38 Higgins JPT, Green S. Cochrane Handbook for Systematic Reviews of Interventions. John Wiley \& Sons 2011. 